\title{
Interacting galaxies in 3D: Three case studies.
}

\author{
Isaura Fuentes-Carrera ${ }^{1}$, Nelli Cárdenas-Martínez ${ }^{1}$, \\ Mónica Sánchez-Cruces ${ }^{1}$, Margarita Rosado ${ }^{2}$, Philippe Amram ${ }^{3}$, \\ Lorenzo Olguín ${ }^{4}$, Jura Borissova ${ }^{5}$, Simon Verley ${ }^{6}$, Héctor Flores ${ }^{7}$, \\ Lourdes Verdes-Montenegro ${ }^{8}$ and Denise Gonçalves ${ }^{9}$ \\ ${ }^{1}$ Escuela Superior de Física y Matemáticas, Instituto Politécnico Nacional (ESFM-IPN) \\ Edificio 9, U. P. Adolfo López Mateos s/n, Col. Zacatenco, 07730 Mexico City, Mexico \\ email: isaura@esfm.ipn.mx \\ ${ }^{2}$ IA-UNAM, Mexico ${ }^{3}$ LAM, France ${ }^{4}$ UNISON, Mexico ${ }^{5}$ Universidad de Valparaíso, Chile \\ ${ }^{6}$ Universidad de Granada, Spain ${ }^{7}$ Observatoire de Paris-Meudon, France ${ }^{8}$ IAA, Spain \\ ${ }^{9}$ Observatorio do Valongo, Rio de Janeiro, Brazil
}

\begin{abstract}
We present 3D observations of three interacting galaxies in order derive their extended kinematics and to trace events such as violent star-formation (SF), mass-transfer, structure perturbation and the presence of energetic sources induced by the interacting processes.
\end{abstract}

Keywords. galaxies: interacting - galaxies: spiral - galaxies: evolution

\section{Introduction}

External perturbations and induced star formation. We present scanning Fabry-Perot interferometric observations of a pair of starburst galaxies. We derived the velocity field of each galaxy and compared them with morphologicial features related to SF phenomena.

Kinematics of an "isolated" galaxy. We also study the kinematics of an apparently isolated LIRG galaxy. We find double $\mathrm{H} \alpha$ profiles that could be related to an infalling satellite triggering an intense SF event.

Energetic phenomena in interacting galaxies. Finally we present an IFU study of the surroundings of an elongated ultra-luminous X-ray source in an interacting galaxy pair (Rosado, Ghosh \& Fuentes-Carrera 2008) in order to trace particular motions of the ionized gas around this source.

\section{Conclusions}

3D observations of galaxies enable us to match the motions of stars and gas with different morphological structures. In the case of interacting galaxies these studies allow us to trace the effects of the interacting process in the evolution of these systems.

\section{Acknowledgements}

I.F-C. acknowledges financial support from CONACYT project 133520 and SIP project 20144432.

\section{References}

Rosado, M., Ghosh, K., \& Fuentes-Carrera, I. 2008, AJ, 136, 212 